\title{
TOPOLOGICAL SENSITIVITY ANALYSIS FOR THE ANISOTROPIC LAPLACE PROBLEM
}

\author{
IMEN KALLEL ${ }^{1,2, *}$ \\ ${ }^{1}$ Northern Border University, College of Science, Arar, P.O. Box 1631, Saudi Arabia \\ ${ }^{2}$ UR Analysis and Control of PDE's, UR 13E64, Department of Mathematics, Faculty of Sciences of \\ Monastir, University of Monastir, 5019 Monastir, Tunisie \\ *Corresponding author: imenkallel16@gmail.com
}

\begin{abstract}
This paper is concerned with the reconstruction of objects immersed in anisotropic media from boundary measurements. The aim of this paper is to propose an alternative approach based on the KohnVogelius formulation and the topological sensitivity analysis method. The idea is to formulate the reconstruction problem as a topology optimization one minimizing an energy-like function. We derive a topological asymptotic expansion for the anisotropic Laplace operator. The unknown object is reconstructed using level-set curve of the topological gradient. We make finally some numerical examples proving the efficiency and accuracy of the proposed algorithm.
\end{abstract}

\section{INTRODUCTION}

In this work we will establish a topological sensitivity analysis for the anisotropic Laplace operator. The topological sensitivity analysis consists of studying the variation of a given cost functional with respect to the presence of a small domain perturbation, such as the insertion of inclusions, cavities, cracks or source-terms. In our paper we concentrate in a small Dirichlet geometric perturbation.

Let us briefly discuss the history of this method. Its main idea was originally introduced by Schumacher [22] in the context of compliance minimization in linear elasticity. In the same context Sokolowski and

Received August $28^{\text {th }}, 2021$; accepted October 12 ${ }^{\text {th }}, 2021$; published November $16^{\text {th }}, 2021$.

2010 Mathematics Subject Classification. 49Q12, 65N21, 35N10.

Key words and phrases. topological gradient; Kohn-Vogelius formulation; Laplace problem; reconstruction problem.

(C)2021 Authors retain the copyrights of their papers, and all open access articles are distributed under the terms of the Creative Commons Attribution License. 
Zochowski [13], who studied the effect of an extract infinitesimal part of the material in structural mechanics. Then in [17] Masmoudi worked out a topological sensitivity analysis framework based on a generalization of the adjoint method and on the use of a truncation technique. By using this framework the topological sensitivity is obtained for several equations $[8,18,20,21]$. For other works on the topological sensitivity concept, we refer to the book by Novotny and Sokolowski [19].

The general idea of the proposed method is to rephrase the inverse problem as a topology optimization problem, where the object immersed in the anisotropic media is the unknown variable. Our aim is to detect this unknown object immersed from over-determined boundary data.

Let $\mathcal{H}$ be an unknown object immersed inside the background domain $\Omega$ and having a smooth boundary $\Sigma=\partial \mathcal{H}$. The geometric inverse problem that we consider here can be formulated as follows:

- Giving two boundary data on $\Gamma$; an imposed flux $\Phi \in H^{-1 / 2}(\Gamma)$ and a measured datum $\varphi_{d} \in$ $H^{1 / 2}(\Gamma)$.

- Find the unknown location of the object $\mathcal{H}$ inside the domain $\Omega$ such that the solution $\varphi$ of the anisotropic Laplace equation satisfies the following over-determined boundary value problem

$$
\begin{cases}-\operatorname{div}(A \nabla \varphi)=\mathcal{F} & \text { in } \quad \Omega \backslash \overline{\mathcal{H}} \\ A \nabla \varphi \cdot \mathbf{n}=\Phi & \text { on } \quad \Gamma, \\ \varphi=\varphi_{d} & \text { on } \quad \Gamma, \\ \varphi=0 & \text { on } \quad \partial \mathcal{H} .\end{cases}
$$

where $A$ is a symmetric positive definite matrix, $\mathbf{n}$ is the exterior unit normal vector and $\mathcal{F} \in L^{2}(\Omega)$ is a given source term.

In this formulation the domain $\Omega \backslash \overline{\mathcal{H}}$ is unknown since the free boundary $\sum$ is unknown. This problem is ill-posed in the sense of Hadamard [10]. The majority of works dealing with this kind of problems fall into the category of shape optimization and based on the shape differentiation technics. It is proved in $[3,7]$ that the studied inverse problems, treated as a shape optimization problems, are severely ill-posed (i.e. unstable), for both Dirichlet and Neumann conditions on the boundary $\sum$. Thus they have to use some regularization methods to solve them numerically.

To solve this inverse problem, we extend the topological sensitivity analysis notion to the anisotropic case and we suggest an alternative approach based on the Kohn-Vogelius formulation [6] and the topological gradient method $[1,2,5,8,9,16,17]$. We combine here the advantages of the Kohn-Vogelius formulation as a self regularization technique and the topological gradient approach as an accurate and fast method.

The first step of our approach is based on the Kohn-Vogelius formulation which rephrase the considered geometrical inverse problem into a topology optimization one. It leads to define for any given permutation 
$\mathcal{H}$ two forward problems: The first one, called Neumann problem, is associated to the Neumann datum $\Phi$

$$
\left(\mathcal{P}_{\mathcal{N}}\right) \begin{cases}-\operatorname{div}\left(A \nabla \varphi_{N}\right)=\mathcal{F} & \text { in } \quad \Omega \backslash \overline{\mathcal{H}} \\ A \nabla \varphi_{N} \cdot \mathbf{n}=\Phi & \text { on } \quad \Gamma \\ \varphi_{N}=0 & \text { on } \quad \partial \mathcal{H} .\end{cases}
$$

The second one is associated to the measured data $\varphi_{d}$, it is called the Dirichlet problem:

$$
\left(\mathcal{P}_{\mathcal{D}}\right) \begin{cases}-\operatorname{div}\left(A \nabla \varphi_{D}\right)=\mathcal{F} & \text { in } \quad \Omega \backslash \overline{\mathcal{H}} \\ \varphi_{D}=\varphi_{d} & \text { on } \quad \Gamma \\ \varphi_{D}=0 & \text { on } \quad \partial \mathcal{H} .\end{cases}
$$

One can observe that if $\mathcal{H}$ coincides with the exact obstacle $\mathcal{H}^{*}$, the misfit between the solutions of $\left(\mathcal{P}_{\mathcal{N}}\right)$ and $\left(\mathcal{P}_{\mathcal{D}}\right)$ vanishes: $\varphi_{N}=\varphi_{D}$. Starting from this observation, the inverse problem can be formulated as a topological optimization one. The unknown object will be characterized as the minimum of the following Kohn-Vogelius type functional [6]

$$
\mathcal{J}(\Omega \backslash \overline{\mathcal{H}})=\int_{\Omega \backslash \overline{\mathcal{H}}} A\left|\nabla \varphi_{N}(x)-\nabla \varphi_{D}(x)\right|^{2} d x
$$

Where the Kohn-Vogelius function is exactly

$$
\mathcal{J}(\Omega \backslash \overline{\mathcal{H}})=\int_{\Omega \backslash \overline{\mathcal{H}}}\left[\nabla \varphi_{N}(x)-\nabla \varphi_{D}(x)\right]^{T} \cdot A\left[\nabla \varphi_{N}(x)-\nabla \varphi_{D}(x)\right] d x
$$

More precisely, the identification problem can be formulated as follow:

$$
(\mathcal{P})\left\{\begin{array}{c}
\text { Find } \mathcal{H}^{*} \subset \Omega \text { such that } \\
\mathcal{J}\left(\Omega \backslash \overline{\mathcal{H}^{*}}\right)=\min _{\mathcal{H} \in D_{a d}} \mathcal{J}(\Omega \backslash \overline{\mathcal{H}})
\end{array}\right.
$$

where $D_{a d}$ is a given set of admissible domains.

To solve the topological optimization problem $(\mathcal{P})$ and detect the location of the unknown object we will derive a topological sensitivity analysis for the Kohn-Vogelius function $\mathcal{J}$ which gives the variation of a criterion with respect to the presence of a small Dirichlet geometric perturbation in the domain. A one-shot reconstruction algorithm is proposed. The main advantage of this algorithm is that, it provides fast and accurate results for detection.

The paper is organized as follows. In the next Section, we present the perturbed Neumann and Dirichlet problems. In Section 3 we study the topological sensitivity analysis for the function $\mathcal{J}$. The obtained results are based on a preliminary estimate describing the perturbation caused by the presence of a small geometry modification of the background domain $\Omega$. A simplified formulation of the shape function variation with respect to the creation of the hole $\mathcal{H}_{z, \varepsilon}$ in $\Omega$ is derived in Section 4 . The Section 5 is devoted to the KohnVogelius type function variation. The proposed numerical algorithm and the detection results are described in Section 6. 


\section{The PERTURBed PROBlems}

In this section, we present the Neumann and Dirichlet problems in the perturbed domain. In the presence of a small geometry perturbation $\mathcal{H}_{z, \varepsilon}$ inside the domain $\Omega$, the Neumann problem consists in finding $\varphi_{N}^{\varepsilon} \in H^{1}\left(\Omega \backslash \overline{\mathcal{H}_{z, \varepsilon}}\right)$ solution to

$$
\begin{cases}-\operatorname{div}\left(A \nabla \varphi_{N}^{\varepsilon}\right)=\mathcal{F} & \text { in } \quad \Omega \backslash \overline{\mathcal{H}_{z, \varepsilon}}, \\ A \nabla \varphi_{N}^{\varepsilon} \cdot \mathbf{n}=\Phi & \text { on } \quad \Gamma_{n}, \\ \varphi_{N}^{\varepsilon}=0 & \text { on } \quad \partial \mathcal{H}_{z, \varepsilon} .\end{cases}
$$

The Neumann problem in the non perturbed domain is: find $\varphi_{N}^{0} \in H^{1}(\Omega)$ solution to

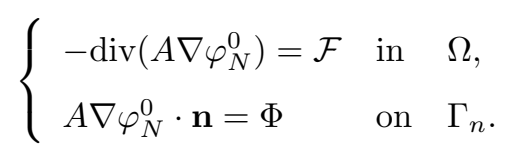

Similarly, the perturbed Dirichlet problem consists in finding $\varphi_{D}^{\varepsilon} \in H^{1}\left(\Omega \backslash \overline{\mathcal{H}_{z, \varepsilon}}\right)$ solution to

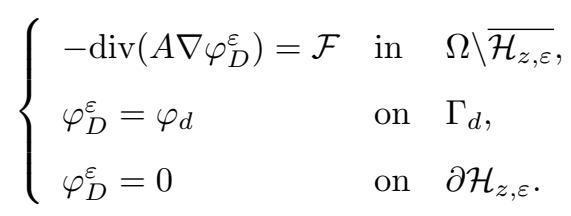

In the absence of any perturbation (i.e $\varepsilon=0)$, the Dirichlet problem is: find $\varphi_{D}^{0} \in H^{1}(\Omega)$ solution to

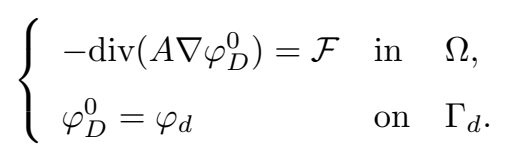

We introduce the considered shape functional $\mathcal{J}$. Given a small geometrical perturbation $\mathcal{H}_{z, \varepsilon}$ inside the initial domain $\Omega$, the function $\mathcal{J}$ measures the difference between the Neumann and Dirichlet perturbed solutions. We define $\mathcal{J}$ as

$$
\mathcal{J}\left(\Omega \backslash \overline{\mathcal{H}_{z, \varepsilon}}\right)=\int_{\Omega \backslash \overline{\mathcal{H}} z, \varepsilon} A\left|\nabla \varphi_{N}^{\varepsilon}(x)-\nabla \varphi_{D}^{\varepsilon}(x)\right|^{2} d x, \forall \mathcal{H}_{z, \varepsilon} \subset \Omega
$$

In the non perturbed domain $(\varepsilon=0)$, the function $\mathcal{J}$ is expressed as

$$
\mathcal{J}(\Omega)=\int_{\Omega} A\left|\nabla \varphi_{N}^{0}(x)-\nabla \varphi_{D}^{0}(x)\right|^{2} d x .
$$

Our aim is to derive an asymptotic expansion for the function $\mathcal{J}$ and calculate the topological sensitivity function $\delta \mathcal{J}$.

The variation of the function $\mathcal{J}$ with respect to the presence of a small perturbation is given by

$$
\begin{aligned}
\mathcal{J}\left(\Omega \backslash \overline{\mathcal{H}_{z, \varepsilon}}\right)-\mathcal{J}(\Omega) & =\int_{\Omega \backslash \overline{\mathcal{H}_{z, \varepsilon}}} A\left|\nabla \varphi_{N}^{\varepsilon}(x)-\nabla \varphi_{D}^{\varepsilon}(x)\right|^{2} d x \\
& -\quad \int_{\Omega} A\left|\nabla \varphi_{N}^{0}(x)-\nabla \varphi_{D}^{0}(x)\right|^{2} d x .
\end{aligned}
$$

In the following, we will derive a topological sensitivity analysis valid for all function $\mathcal{J}_{\varepsilon}$ verifying the following hypothesis: 
Hypothesis 2.1. - The function $\mathcal{J}_{0}$ is differentiable in $H^{1}(\Omega)$.

- There exist a real number $\delta \mathcal{J} \in \mathbb{R}$, independent of $\varepsilon$ and a scalar function $\rho: \mathbb{R}_{+} \rightarrow \mathbb{R}_{+}$such that $\forall \varepsilon \geq 0$

$$
\begin{gathered}
\mathcal{J}_{\varepsilon}\left(\varphi_{\varepsilon}\right)-\mathcal{J}_{0}\left(\varphi_{0}\right)=D \mathcal{J}_{0}\left(\varphi_{0}\right)\left(\varphi_{\varepsilon}-\varphi_{0}\right)+\rho(\varepsilon) \delta \mathcal{J}+o(\rho(\varepsilon)), \\
\lim _{\varepsilon \rightarrow 0} \rho(\varepsilon)=0
\end{gathered}
$$

with $\varphi_{\varepsilon}$ is the solution of the perturbed anisotropic Laplace problem inside the perturbed domain $\Omega \backslash \overline{\mathcal{H}_{z, \varepsilon}}$

$$
\left(\mathcal{P}_{\varepsilon}\right) \begin{cases}-\operatorname{div}\left(A \nabla \varphi_{\varepsilon}\right)=\mathcal{F} & \text { in } \quad \Omega \backslash \overline{\mathcal{H}_{z, \varepsilon}}, \\ A \nabla \varphi_{\varepsilon} \cdot \mathbf{n}=\Phi & \text { on } \Gamma, \\ \varphi_{\varepsilon}=\varphi_{d} & \text { on } \Gamma, \\ \varphi_{\varepsilon}=0 & \text { on } \quad \partial \mathcal{H}_{z, \varepsilon} .\end{cases}
$$

Here $\delta \mathcal{J}$ is called the topological sensitivity function.

\section{Topological ASYMPtotic EXPANSION}

In this section, we derive a topological asymptotic expansion for the anisotropic Laplace operator. We start our analysis by establishing a variational formulation associated to the anisotropic Laplace system. From the weak formulation of 2.1, we deduce that $\varphi_{\varepsilon} \in \mathcal{V}_{\varepsilon}$ is the unique solution to

$$
a_{\varepsilon}\left(\varphi_{\varepsilon}, v\right)=l_{\varepsilon}(v) \quad \forall v \in \mathcal{V}_{\varepsilon}
$$

where the function space $\mathcal{V}_{\varepsilon}$, the bilinear form $a_{\varepsilon}$ and the linear form $l_{\varepsilon}$ are defined by

$$
\mathcal{V}_{\varepsilon}\left\{w \in H^{1}\left(\Omega \backslash \overline{\mathcal{H}_{z, \varepsilon}}\right) ; \quad w=0 \quad \text { in } \quad \Gamma_{d} \cup \partial \mathcal{H}_{z, \varepsilon}\right\}
$$

and for all $\varphi, v \in \mathcal{V}_{\varepsilon}$

$$
\left\{\begin{array}{l}
a_{\varepsilon}(v, w)=\int_{\Omega \backslash \overline{\mathcal{H}_{z, \varepsilon}}}[\nabla w]^{T} \cdot A \nabla v d x, \\
l_{\varepsilon}(w)=\int_{\Omega \backslash \overline{\mathcal{H}_{z, \varepsilon}}} \mathcal{F} w d x+\int_{\Gamma_{n}} \Phi w d s
\end{array}\right.
$$

Under the hypothesis 2.1 , the variation of the shape function $\mathcal{J}$ reads

$$
\begin{aligned}
\mathcal{J}\left(\Omega \backslash \overline{\mathcal{H}_{z, \varepsilon}}\right)-\mathcal{J}(\Omega) & =\mathcal{J}_{\varepsilon}\left(\varphi_{\varepsilon}\right)-\mathcal{J}_{0}\left(\varphi_{0}\right) \\
& =D \mathcal{J}_{0}\left(\varphi_{0}\right)\left(\varphi_{\varepsilon}-\varphi_{0}\right)+\rho(\varepsilon) \delta \mathcal{J}+o(\rho(\varepsilon))
\end{aligned}
$$

Let $v_{0} \in \mathcal{V}_{0}$ be the solution to the associated adjoint problem

$$
a_{0}\left(w, v_{0}\right)=D \mathcal{J}_{0}\left(\varphi_{0}\right)(w), \forall w \in \mathcal{V}_{0}
$$


Then, the shape function variation rewritten as

$$
\mathcal{J}\left(\Omega \backslash \overline{\mathcal{H}_{z, \varepsilon}}\right)-\mathcal{J}(\Omega)=a_{0}\left(\varphi_{0}-\varphi_{\varepsilon}, v_{0}\right)+\rho(\varepsilon) \delta \mathcal{J}+o(\rho(\varepsilon))
$$

Aiming to derive an asymptotic expansion for $\mathcal{J}$, we examine in the next section the asymptotic behavior with respect to $\varepsilon$ of the term $a_{0}\left(\varphi_{0}-\varphi_{\varepsilon}, v_{0}\right)$.

3.1. Asymptotic formula for the anisotropic Laplace problem. This section is devoted to the main theoretical result. A topological asymptotic expansion is derived for the anisotropic Laplace operator with respect to the presence of a small topological perturbation $\mathcal{H}_{z, \varepsilon}$ in the initial domain. The obtained results are general and valid for large class of cost functions. More precisely, the derived asymptotic expansion is valid for all cost function $\mathcal{J}$ satisfying the assumption 2.1 .

The main result of this section is summarized in the following theorem.

Theorem 3.1. Let $j$ a design function of the form $j\left(\Omega \backslash \overline{\mathcal{H}}(, \varepsilon)=\mathcal{J}\left(\varphi_{\varepsilon}\right)\right.$. If $\mathcal{J}$ satisfies the assumption 2.1 , then $j$ has the following asymptotic expansion

$$
j\left(\Omega \backslash \overline{\mathcal{H}_{z, \varepsilon}}\right)-j(\Omega)=\rho(\varepsilon)(\delta a+\delta \mathcal{J})+o(\rho(\varepsilon))
$$

where

$$
\begin{cases}\rho(\varepsilon)=\frac{-1}{\log (\varepsilon)}, & \delta a=2 \pi \sqrt{|A|} \varphi_{0}(z) v_{0}(z) \quad \text { if } \quad d=2, \\ \rho(\varepsilon)=\varepsilon, & \delta a=-4 \pi \sqrt{|A|} \varphi_{0}(z) v_{0}(z) \quad \text { if } \quad d=3 .\end{cases}
$$

The term $\delta \mathcal{J}$ is the variation of the considered cost function $\mathcal{J}$.

In order to check the hypothesis 2.1 , we derive an asymptotic expansion the variation of the bilinear form. We have

$$
a_{0}\left(\varphi_{0}-\varphi_{\varepsilon}, v_{0}\right)=\int_{\Omega}\left[\nabla\left(\varphi_{0}-\varphi_{\varepsilon}\right)\right]^{T} \cdot A \nabla v_{0} d x
$$

Using the Green formula, we obtain

$$
a_{0}\left(\varphi_{0}-\varphi_{\varepsilon}, v_{0}\right)=\int_{\mathcal{H}_{z, \varepsilon}}\left[\nabla \varphi_{0}\right]^{T} \cdot A \nabla v_{0} d x+\int_{\partial \mathcal{H}_{z, \varepsilon}}\left[A \nabla\left(\varphi_{0}-\varphi_{\varepsilon}\right)\right]^{T} \cdot \mathbf{n} v_{0} d x
$$

Next, we shall examine each term on the right hand side of 3.2 separately. The following lemma gives an estimate for the first term.

Lemma 3.1. We have

$$
\int_{\mathcal{H}_{z, \varepsilon}}\left[\nabla \varphi_{0}\right]^{T} \cdot A \nabla v_{0} d x=\varepsilon^{d}|\mathcal{H}|\left[\nabla \varphi_{0}(z)\right]^{T} \cdot A \nabla v_{0}(z)+o\left(\varepsilon^{d}\right)
$$




\section{Proof}

Using the change of variable $x=z+\varepsilon y$ one obtains

$$
\begin{aligned}
\int_{\mathcal{H}_{z, \varepsilon}}\left[\nabla \varphi_{0}\right]^{T} \cdot A \nabla v_{0} d x & =\varepsilon^{d}|\mathcal{H}|\left[\nabla \varphi_{0}(z)\right]^{T} \cdot A \nabla v_{0}(z) \\
& +\varepsilon^{d} \int_{\mathcal{H}}\left\{\left[\nabla \varphi_{0}(x+\varepsilon y)\right]^{T} \cdot A \nabla v_{0}(z+\varepsilon y)-\left[\nabla \varphi_{0}(z)\right]^{T} \cdot A \nabla v_{0}(z)\right\} d y
\end{aligned}
$$

Due the the smoothness of $\varphi_{0}$ and $v_{0}$ in $\mathcal{H}_{z, \varepsilon}$, we derive

$$
\lim _{\varepsilon \rightarrow 0} \int_{\mathcal{H}}\left\{\left[\nabla \varphi_{0}(x+\varepsilon y)\right]^{T} \cdot A \nabla v_{0}(z+\varepsilon y)-\left[\nabla \varphi_{0}(z)\right]^{T} \cdot A \nabla v_{0}(z)\right\} d y=0
$$

Then it follows

$$
\int_{\mathcal{H}_{z, \varepsilon}}\left[\nabla \varphi_{0}\right]^{T} \cdot A \nabla v_{0} d x=\varepsilon^{d}|\mathcal{H}|\left[\nabla \varphi_{0}(z)\right]^{T} \cdot A \nabla v_{0}(z)+o\left(\varepsilon^{d}\right) .
$$

Next to examine the second term of 3.2 we introduce the variable

$$
\chi_{\varepsilon}=\varphi_{0}-\varphi_{\varepsilon} .
$$

it is easily to show that $\chi_{\varepsilon}$ satisfies the following system

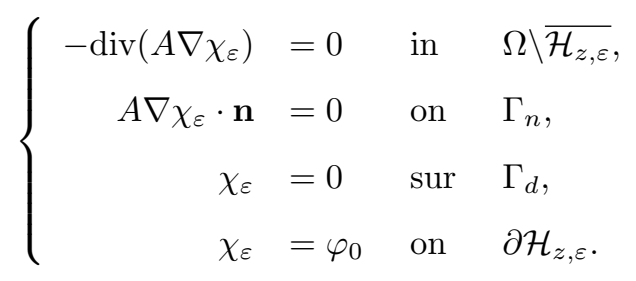

We can write $\chi_{\varepsilon}$ as

$$
\chi_{\varepsilon}=h_{\varepsilon}+r_{\varepsilon}
$$

where

$$
h_{\varepsilon}(x)=\frac{E(x-z)}{E(z)} \varphi_{0}(z), \quad x \in \Omega,
$$

with $E$ is the fundamental solution of the anisotropic Laplace operator [14]:

$$
E(x)= \begin{cases}\frac{1}{2 \pi \sqrt{|A|}} \log \left(\| A_{*} x||\right), & d=2, \\ \frac{-1}{4 \pi \sqrt{|A| \mid}\left|A_{*} x\right| \mid}, & d=3 .\end{cases}
$$


where $|A|$ is the determinant of $A, A_{*}$ be the positive-definite symmetric matrix such that $A_{*}^{2}=A^{-1}$.

Then $r_{\varepsilon}$ is solution of

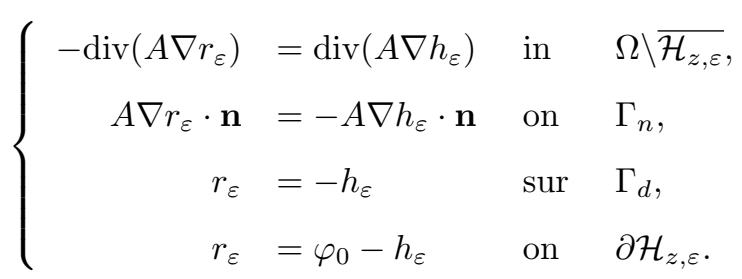

We set

$$
\begin{aligned}
& R_{1}(\varepsilon)=\int_{\partial \mathcal{H}_{z, \varepsilon}}\left[A \nabla h_{\varepsilon}\right]^{T} \cdot \mathbf{n}\left(v_{0}-v_{0}(z)\right) d s \\
& R_{2}(\varepsilon)=\int_{\partial \mathcal{H}_{z, \varepsilon}}\left[A \nabla r_{\varepsilon}\right]^{T} \cdot \mathbf{n} v_{0} d s
\end{aligned}
$$

Lemma 3.2. We have

$$
\int_{\partial \mathcal{H}_{z, \varepsilon}}\left[A \nabla\left(\varphi_{0}-\varphi_{\varepsilon}\right)\right]^{T} \cdot \mathbf{n} v_{0} d s=-\frac{\varphi_{0}(z) v_{0}(z)}{E(\varepsilon)}+R_{1}(\varepsilon)+R_{2}(\varepsilon)
$$

\section{Proof}

Using the Green formulation, we obtain

$$
\begin{aligned}
\int_{\partial \mathcal{H}_{z, \varepsilon}}\left[A \nabla\left(\varphi_{0}-\varphi_{\varepsilon}\right)\right]^{T} \cdot \mathbf{n} v_{0} d s & =\int_{\partial \mathcal{H}_{z, \varepsilon}}\left[A \nabla \chi_{\varepsilon}\right]^{T} \cdot \mathbf{n} v_{0} d s \\
& =\int_{\partial \mathcal{H}_{z, \varepsilon}}\left[A \nabla h_{\varepsilon}\right]^{T} \cdot \mathbf{n} v_{0} d s+\int_{\partial \mathcal{H}_{z, \varepsilon}}\left[A \nabla r_{\varepsilon}\right]^{T} \cdot \mathbf{n} v_{0} d s \\
& =\int_{\partial \mathcal{H}_{z, \varepsilon}}\left[A \nabla h_{\varepsilon}\right]^{T} \cdot \mathbf{n}\left(v_{0}-v_{0}(z)\right) d s \\
& +\int_{\partial \omega_{\varepsilon}}\left[A \nabla h_{\varepsilon}\right]^{T} \cdot \mathbf{n} v_{0}(z) d s+R_{2}(\varepsilon) \\
& =\int_{\partial \mathcal{H}_{z, \varepsilon}}\left[A \nabla h_{\varepsilon}\right]^{T} \cdot \mathbf{n} v_{0}(z) d s+R_{1}(\varepsilon)+R_{2}(\varepsilon) \\
& =\frac{\varphi_{0}(z) v_{0}(z)}{E(\varepsilon)} \int_{\partial \omega_{\varepsilon}}[A \nabla E(x-z)]^{T} \cdot \mathbf{n} d s+R_{1}(\varepsilon)+R_{2}(\varepsilon) .
\end{aligned}
$$

Such that

$$
\int_{\partial \mathcal{H}_{z, \varepsilon}}[A \nabla E(x-z)]^{T} \cdot \mathbf{n} d s=-1
$$

Hence

$$
\int_{\mathcal{H}_{z, \varepsilon}}\left[\nabla \chi_{\varepsilon}\right]^{T} \cdot A \nabla v_{0} d x=-\frac{\varphi_{0}(z) v_{0}(z)}{E(\varepsilon)}+R_{1}(\varepsilon)+R_{2}(\varepsilon)
$$

Assuming that $R_{i}(\varepsilon)=o(\rho(\varepsilon)), i=1,2$. We will give the proof for the two dimensional case in section 4.3.

Besides thanks to the fundamental solution, we obtain the main result presented in the following sections concerns the topological asymptotic expansion of an arbitrary design function $j$. Some cost function examples are presented in section 4 . 


\section{A particular Class of COSt FunCtion}

In this section, we present some useful examples of shape functions and we gives their variations $\delta \mathcal{J}$.

4.1. First example. This example is concerned with the $L^{2}-$ norm. We consider the shape function defined by

$$
\mathcal{J}\left(\Omega \backslash \overline{\mathcal{H}_{z, \varepsilon}}\right)=\int_{\Omega \backslash \overline{\mathcal{H}_{z, \varepsilon}}}\left|\varphi_{\varepsilon}-\varphi_{d}\right|^{2} d x, \forall \varphi_{\varepsilon} \in H^{1}\left(\Omega \backslash \overline{\mathcal{H}_{z, \varepsilon}}\right)
$$

where $\varphi_{d} \in H^{1}(\Omega)$ is a given desired (objective) function.

Proposition 4.1. The cost function $\mathcal{J}_{\varepsilon}$ defined by

$$
\mathcal{J}_{\varepsilon}(\varphi)=\int_{\Omega \backslash \overline{\mathcal{H}_{z, \varepsilon}}}\left|\varphi-\varphi_{d}\right|^{2} d x, \forall \varphi \in H^{1}\left(\Omega \backslash \overline{\mathcal{H}_{z, \varepsilon}}\right)
$$

satisfies the hypothesis 2.1 with

$$
D \mathcal{J}_{0}\left(\varphi_{0}\right)=2 \int_{\Omega}\left(\varphi_{0}-\varphi_{d}\right) w d x, \forall w \in H^{1}(\Omega) \quad \text { and } \quad \delta \mathcal{J}(x)=0, \forall x \in \Omega
$$

4.2. Second example. Here, we are dealing with the $H^{1}$-semi-norm. We consider the shape function

$$
\mathcal{J}\left(\Omega \backslash \overline{\mathcal{H}_{z, \varepsilon}}\right)=\int_{\Omega \backslash \overline{\mathcal{H}_{z, \varepsilon}}} A\left|\nabla \varphi_{\varepsilon}-\nabla \varphi_{d}\right|^{2} d x
$$

with $\varphi_{d} \in H^{2}(\Omega)$ is a given desired function.

Proposition 4.2. The cost function $\mathcal{J}_{\varepsilon}$ defined by

$$
\mathcal{J}_{\varepsilon}(\varphi)=\int_{\Omega \backslash \overline{\mathcal{H}_{z, \varepsilon}}} A\left|\nabla \varphi-\nabla \varphi_{d}\right|^{2} d x, \forall \varphi \in H^{1}\left(\Omega \backslash \overline{\mathcal{H}_{z, \varepsilon}}\right)
$$

satisfies the hypothesis 2.1 with

$$
D \mathcal{J}_{0}\left(\varphi_{0}\right)=2 \int_{\Omega}\left(\nabla \varphi_{0}-\nabla \varphi_{d}\right)^{T} \cdot A \nabla w d x, \forall w \in H^{1}(\Omega)
$$

and

$$
\forall x \in \Omega, \quad \delta \mathcal{J}(x)= \begin{cases}2 \pi \sqrt{|A|}\left|\varphi_{0}(x)\right|^{2}, & d=2 \\ -4 \pi \sqrt{|A|}\left|\varphi_{0}(x)\right|^{2}, & d=3 .\end{cases}
$$

\subsection{Proofs.}


4.3.1. Preliminary results. The aim of this section is to give some technical results which will be used in section 4.3 .

Lemma 4.1. [4] Consider $\psi \in H^{1 / 2}\left(\partial \mathcal{H}_{z, \varepsilon}\right), g_{d} \in H^{1 / 2}\left(\Gamma_{d}\right)$ and $g_{n} \in H^{-1 / 2}\left(\Gamma_{n}\right)$

If $X$ is solution of the following system

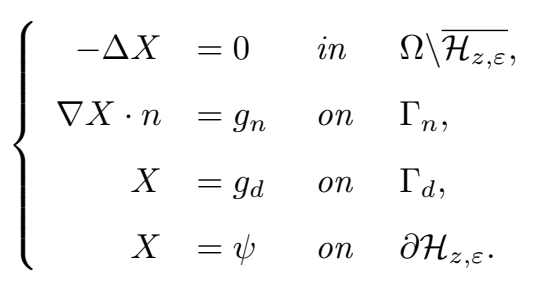

There exists a non negative constant $c$ such that

$$
\|X\|_{1, \Omega \backslash \overline{\mathcal{H}_{z, \varepsilon}}} \leq c\left[\frac{1}{\sqrt{-\log (\varepsilon)}}\|\psi(z+\varepsilon y)\|_{1 / 2, \partial \mathcal{H}}+\left\|g_{d}\right\|_{1 / 2, \Gamma_{d}}+\left\|g_{n}\right\|_{-1 / 2, \Gamma_{n}}\right]
$$

Lemma 4.2. The function defined by $h_{\varepsilon}(x)=\frac{E(x-z)}{E(\varepsilon)} \varphi_{0}(z), \forall x \in \Omega \backslash \overline{\mathcal{H}_{z, \varepsilon}}$ admits the following estimates

$$
\left\|h_{\varepsilon}\right\|_{1, \Omega_{R}} \leq \frac{-c}{\log \varepsilon} \quad\left\|h_{\varepsilon}\right\|_{1, \Omega \backslash \overline{\mathcal{H}} z, \varepsilon} \leq \frac{c}{\sqrt{-\log \varepsilon}}
$$

Where $R$ is a strictly positive real number such that $\omega_{\varepsilon} \subset B(z, R) \subset \Omega$, we set $\Omega_{R}=\Omega \backslash \overline{B(z, R)}$.

Proof of Lemma 4.2

Using the definition of $h_{\varepsilon}$, we have

$$
\left\|h_{\varepsilon}\right\|_{1, \Omega_{R}}=\frac{-1}{\log \varepsilon}\left\|\varphi_{0}(z) \log (|x-z|)\right\|_{1, \Omega_{R}}
$$

Since that $z \notin \Omega_{R}$, we deduce that

$$
\left\|h_{\varepsilon}\right\|_{1, \Omega_{R}} \leq \frac{-c}{\log \varepsilon}
$$

Then we have $h_{\varepsilon}$ is solution of

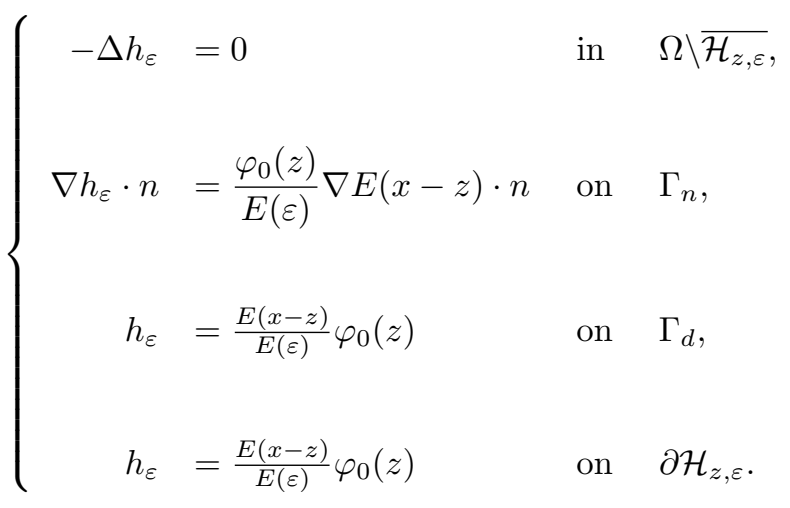


Note that, by Lemma 4.1,

$$
\begin{aligned}
\left\|h_{\varepsilon}\right\|_{1, \Omega \backslash \overline{\mathcal{H}} z, \varepsilon} \leq & c\left\{\frac{1}{\sqrt{-\log (\varepsilon)}}\left\|\frac{E(\varepsilon y)}{E(\varepsilon)} \varphi_{0}(z)\right\|_{\frac{1}{2}, \partial \mathcal{H}}+\left\|\frac{\varphi_{0}(z)}{E(\varepsilon)} \nabla E(x-z) \cdot n\right\|_{\frac{-1}{2}, \Gamma_{n}}\right. \\
& \left.+\left\|\frac{E(x-z)}{E(\varepsilon)} \varphi_{0}(z)\right\|_{\frac{1}{2}, \Gamma_{d}}\right\} \\
\leq & \frac{c_{1}}{\sqrt{-\log (\varepsilon)}}+\frac{c_{2}}{-\log (\varepsilon)} .
\end{aligned}
$$

Then,

$$
\left\|h_{\varepsilon}\right\|_{1, \Omega \backslash \overline{\mathcal{H}_{z, \varepsilon}}} \leq \frac{c}{\sqrt{-\log (\varepsilon)}}
$$

This completes the proof.

Lemma 4.3. There exists a constant $C$ strictly positive such that

$$
\left\|\varphi_{0}-h_{\varepsilon}\right\|_{\frac{1}{2}, \partial \mathcal{H}_{z, \varepsilon}} \leq \frac{-c}{\log (\varepsilon)}
$$

Proof of Lemma 4.3

Using the change of variables $x=z+\varepsilon y$, we obtain

$$
\begin{aligned}
\varphi_{0}(x)-h_{\varepsilon}(x) & =\varphi_{0}(z+\varepsilon y)-h_{\varepsilon}(z+\varepsilon y) \\
& =\varphi_{0}(z+\varepsilon y)-\frac{E(\varepsilon y)}{E(\varepsilon)} \varphi_{0}(z) \\
& =\varphi_{0}(z+\varepsilon y)-\left(1+\frac{E(y)}{E(\varepsilon)}\right) \varphi_{0}(z) .
\end{aligned}
$$

The smoothness of $\varphi_{0}$ and $E$ in $\mathcal{H}_{z, \varepsilon}$ and $\mathcal{H}$ gives that

$$
\varphi_{0}(z+\varepsilon y)-\varphi_{0}(z)=O(\varepsilon)
$$

and

$$
\frac{E(y) \varphi_{0}(z)}{E(\varepsilon)}=O\left(\frac{-1}{\log (\varepsilon)}\right)
$$

Hence

$$
\varphi_{0}(x)-h_{\varepsilon}(x)=O\left(\frac{-1}{\log (\varepsilon)}\right)
$$

Thus the proof is complete.

Lemma 4.4. We have the following estimation

$$
\left\|r_{\varepsilon}\right\|_{1, \Omega \backslash \overline{\mathcal{H}_{z, \varepsilon}}} \leq \frac{c}{\sqrt{-\log (\varepsilon)}} .
$$




\section{Proof of Lemma 4.4}

We can write $r_{\varepsilon}$ solution of the system 3.4 as follows $r_{\varepsilon}=r_{\varepsilon}^{1}+r_{\varepsilon}^{2}$ where $r_{\varepsilon}^{1}$ satisfies

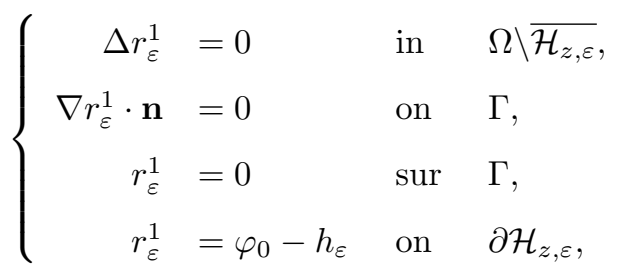

and $r_{\varepsilon}^{2}$ is solution of

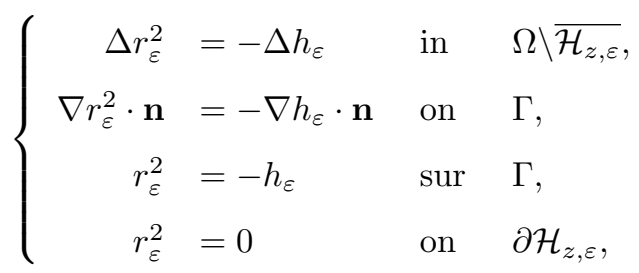

It then follows from 4.3 and the Green formulation that

$$
\int_{\Omega \backslash \overline{\mathcal{H} z, \varepsilon}}\left|\nabla r_{\varepsilon}^{1}\right|^{2} d x=<\nabla r_{\varepsilon}^{1} \cdot \mathbf{n}, \varphi_{0}-h_{\varepsilon}>_{-1 / 2,1 / 2, \partial \mathcal{H}}
$$

Note that, by the theorem of the normal trace, we obtain

$$
\begin{aligned}
\int_{\Omega \backslash \overline{\mathcal{H}_{z, \varepsilon}}}\left|\nabla r_{\varepsilon}^{1}\right|^{2} d x & \leq\left\|\nabla r_{\varepsilon}^{1}\right\|_{-1 / 2, \partial \mathcal{H}_{z, \varepsilon}}\left\|\left(\varphi_{0}-h_{\varepsilon}\right)\right\|_{1 / 2, \partial \mathcal{H}_{z, \varepsilon}} \\
& \leq\left\|r_{\varepsilon}^{1}\right\|_{1, \Omega \backslash \overline{\mathcal{H}_{z, \varepsilon}}}\left\|\varphi_{0}-h_{\varepsilon}\right\|_{1 / 2, \partial \mathcal{H}_{z, \varepsilon}} \\
& \leq \frac{-c}{\log \varepsilon}\left\|r_{\varepsilon}^{1}\right\|_{1, \Omega \backslash \overline{\mathcal{H}_{z, \varepsilon}}}
\end{aligned}
$$

The Poincare inequality gives us

$$
\begin{gathered}
\left\|r_{\varepsilon}^{1}\right\|_{1, \Omega \backslash \overline{\mathcal{H}_{z, \varepsilon}}} \leq c \int_{\Omega \backslash \overline{\mathcal{H}_{z, \varepsilon}}}\left|\nabla r_{\varepsilon}^{1}\right|^{2} d x \\
\left\|r_{\varepsilon}^{2}\right\|_{1, \Omega \backslash \overline{\mathcal{H}_{z, \varepsilon}}} \leq c\left\{\left\|\nabla h_{\varepsilon} \cdot n\right\|_{-1 / 2, \Gamma_{n}}+\left\|h_{\varepsilon}\right\|_{1 / 2, \Gamma_{d}}+\left\|\Delta h_{\varepsilon}\right\|_{0, \Omega \backslash \overline{\mathcal{H}_{z, \varepsilon}}}\right\} \\
\leq\left\|h_{\varepsilon}\right\|_{1, \Omega \backslash \overline{\mathcal{H}_{z, \varepsilon}}}
\end{gathered}
$$

Then,

$$
\left\|r_{\varepsilon}^{2}\right\|_{1, \Omega \backslash \overline{\mathcal{H} z, \varepsilon}} \leq \frac{c}{\sqrt{-\log \varepsilon}}
$$

Since

$$
\left\|r_{\varepsilon}\right\|_{1, \Omega \backslash \overline{\mathcal{H}_{z, \varepsilon}}} \leq\left\|r_{\varepsilon}^{1}\right\|_{1, \Omega \backslash \overline{\mathcal{H}} z, \varepsilon}+\left\|r_{\varepsilon}^{2}\right\|_{1, \Omega \backslash \overline{\mathcal{H}_{z, \varepsilon}}}
$$

We conclude that

$$
\left\|r_{\varepsilon}\right\|_{1, \Omega \backslash \overline{\mathcal{H}_{z, \varepsilon}}} \leq \frac{c}{\sqrt{-\log \varepsilon}}
$$




\section{Proof of theorem 3.1}

We only need to prove that

$$
R_{1}(\varepsilon)=o\left(\frac{-1}{\log (\varepsilon)}\right) \quad \text { and } \quad R_{2}(\varepsilon)=o\left(\frac{-1}{\log (\varepsilon)}\right) .
$$

Remember that

$$
R_{1}(\varepsilon)=\int_{\partial \mathcal{H}_{z, \varepsilon}}\left[A \nabla h_{\varepsilon}\right]^{T} \cdot \mathbf{n}\left(v_{0}-v_{0}(z)\right) d s
$$

We have

$$
\begin{aligned}
\left|R_{1}(\varepsilon)\right| & \leq\|A\|_{\infty}\left\|\nabla h_{\varepsilon} \cdot \mathbf{n}\right\|_{\frac{-1}{2}, \partial \mathcal{H}_{z, \varepsilon}}\left\|v_{0}-v_{0}(z)\right\|_{\frac{1}{2}, \partial \mathcal{H}_{z, \varepsilon}} \\
& \leq\|A\|_{\infty}\left\|h_{\varepsilon}\right\|_{1, \Omega \backslash \overline{\mathcal{H}_{z, \varepsilon}}}\left\|v_{0}-v_{0}(z)\right\|_{\frac{1}{2}, \partial \mathcal{H}_{z, \varepsilon}}
\end{aligned}
$$

Changing variables $x=z+\varepsilon y$ and using the lemma 4.2

$$
\left|R_{1}(\varepsilon)\right| \leq \frac{C \varepsilon}{\sqrt{-\log (\varepsilon)}}=o\left(\frac{-1}{\log (\varepsilon)}\right)
$$

On the other hand

$$
R_{2}(\varepsilon)=\int_{\partial \mathcal{H}_{z, \varepsilon}}\left[A \nabla r_{\varepsilon}\right]^{T} \cdot \mathbf{n} v_{0} d s
$$

We have

$$
\begin{aligned}
\left|R_{2}(\varepsilon)\right| & \leq C|| \nabla r_{\varepsilon}\left\|_{\frac{-1}{2}, \partial \mathcal{H}_{z, \varepsilon}}\right\| v_{0} \|_{\frac{1}{2}, \partial \mathcal{H}_{z, \varepsilon}} \\
& \leq C\left\|r_{\varepsilon}\right\|_{1, \Omega \backslash \overline{\mathcal{H}_{z, \varepsilon}}}\left\|v_{0}\right\|_{1, \mathcal{H}_{z, \varepsilon}} .
\end{aligned}
$$

Likewise, using the same change of variables and due to lemma 4.4, it follows that

$$
\left|R_{2}(\varepsilon)\right| \leq \frac{C \varepsilon}{\sqrt{-\log (\varepsilon)}}=o\left(\frac{-1}{\log (\varepsilon)}\right)
$$

which completes the proof.

\section{Proof of proposition 4.1}

The function $\mathcal{J}$ is differentiable with respect to $\varphi$ and we have

$$
D \mathcal{J}_{0}\left(\varphi_{0}\right)(w)=2 \int_{\Omega}\left(\varphi_{0}-\varphi_{d}\right) w d x, \quad \forall w \in \mathcal{V}_{0}
$$


Computing the variation $\mathcal{J}\left(\Omega \backslash \overline{\mathcal{H}_{z, \varepsilon}}\right)-\mathcal{J}(\Omega)$

$$
\begin{aligned}
\mathcal{J}\left(\Omega \backslash \overline{\mathcal{H}_{z, \varepsilon}}\right)-\mathcal{J}(\Omega) & =\int_{\Omega \backslash \overline{\mathcal{H}}, \varepsilon}\left|\varphi_{\varepsilon}-\varphi_{d}\right|^{2} d x-\int_{\Omega}\left|\varphi_{0}-\varphi_{d}\right|^{2} d x \\
& =\int_{\Omega}\left[\left|\varphi_{\varepsilon}-\varphi_{d}\right|^{2}-\left|\varphi_{0}-\varphi_{d}\right|^{2}\right] d x-\int_{\mathcal{H}_{z, \varepsilon}}\left|\varphi_{d}\right|^{2} d x \\
& =\int_{\Omega}\left[\left|\varphi_{0}-\varphi_{\varepsilon}\right|^{2}-2\left(\varphi_{0}-\varphi_{\varepsilon}\right)\left(\varphi_{0}-\varphi_{d}\right)\right] d x-\int_{\Omega \backslash \overline{\mathcal{H}} z, \varepsilon}\left|\varphi_{d}\right|^{2} d x \\
& =D \mathcal{J}_{0}\left(\varphi_{0}\right)\left(\varphi_{\varepsilon}-\varphi_{0}\right)+\int_{\Omega \backslash \overline{\mathcal{H}_{z, \varepsilon}}}\left|\varphi_{0}-\varphi_{\varepsilon}\right|^{2} d x \\
& +\int_{\mathcal{H}_{z, \varepsilon}}\left|\varphi_{0}\right|^{2} d x-\int_{\mathcal{H}_{z, \varepsilon}}\left|\varphi_{d}\right|^{2} d x .
\end{aligned}
$$

By the divergence formula and the system 3.3, we have

$$
\int_{\Omega \backslash \overline{\mathcal{H}} z, \varepsilon}\left|\varphi_{0}-\varphi_{\varepsilon}\right|^{2} d x=o\left(\frac{-1}{\log (\varepsilon)}\right)
$$

A change of variable and the fact that $\varphi_{0}$ and $\varphi_{d}$ are of class $C^{2}$ in a neighborhood of the origin yield

$$
\int_{\mathcal{H}_{z, \varepsilon}}\left|\varphi_{d}\right|^{2} d x \leq c \varepsilon^{2} \quad \text { and } \quad \int_{\mathcal{H}_{z, \varepsilon}}\left|\varphi_{0}\right|^{2} d x \leq c \varepsilon^{2}
$$

Hence

$$
\mathcal{J}\left(\Omega \backslash \overline{\mathcal{H}_{z, \varepsilon}}\right)-\mathcal{J}(\Omega)=D \mathcal{J}_{0}\left(\varphi_{0}\right)\left(\varphi_{\varepsilon}-\varphi_{0}\right)+o\left(\frac{-1}{\log (\varepsilon)}\right) .
$$

Finally, by theorem 3.1, we deduce

$$
j\left(\Omega \backslash \overline{\mathcal{H}_{z, \varepsilon}}\right)-j(\Omega)=\frac{-1}{\log (\varepsilon)} 2 \pi \sqrt{|A|} \varphi_{0}\left(x_{0}\right) v_{0}\left(x_{0}\right)+o\left(\frac{-1}{\log (\varepsilon)}\right) .
$$

Proof of proposition 4.2

The function $J$ is differentiable and we have

$$
D \mathcal{J}_{0}\left(\varphi_{0}\right)(w)=2 \int_{\Omega} A\left(\nabla \varphi_{0}-\nabla \varphi_{d}\right)^{T} . \nabla w d x, \quad \forall w \in \mathcal{V}_{0}
$$

Moreover, we have

$$
\begin{aligned}
\mathcal{J}\left(\Omega \backslash \overline{\mathcal{H}_{z, \varepsilon}}\right)-\mathcal{J}(\Omega) & =\int_{\Omega \backslash \overline{\mathcal{H}_{z, \varepsilon}}} A\left|\nabla \varphi_{\varepsilon}-\nabla \varphi_{d}\right|^{2} d x-\int_{\Omega} A\left|\nabla \varphi_{0}-\nabla \varphi_{d}\right|^{2} d x \\
& =D \mathcal{J}_{0}\left(\varphi_{0}\right)\left(\varphi_{\varepsilon}-\varphi_{0}\right)+\int_{\mathcal{H}_{z, \varepsilon}} A\left|\nabla \varphi_{0}\right|^{2} d x-\int_{\mathcal{H}_{z, \varepsilon}} A\left|\nabla \varphi_{d}\right|^{2} d x \\
& +\int_{\Omega \backslash \overline{\mathcal{H}_{z, \varepsilon}}} A\left|\nabla \varphi_{0}-\nabla \varphi_{\varepsilon}\right|^{2} d x
\end{aligned}
$$

As $\varphi_{0}$ and $\varphi_{d}$ are sufficiently regular in $\mathcal{H}_{z, \varepsilon}$, we obtain

$$
\int_{\mathcal{H}_{z, \varepsilon}} A\left|\nabla \varphi_{0}\right|^{2} d x=O\left(\varepsilon^{2}\right)=o\left(\frac{-1}{\log (\varepsilon)}\right)
$$


and

$$
\int_{\mathcal{H}_{z, \varepsilon}} A\left|\nabla \varphi_{d}\right|^{2} d x=O\left(\varepsilon^{2}\right)=o\left(\frac{-1}{\log (\varepsilon)}\right) .
$$

By the divergence formula, we have

$$
\begin{aligned}
\int_{\Omega \backslash \overline{\mathcal{H}}_{z, \varepsilon}} A\left|\nabla \varphi_{0}-\nabla \varphi_{\varepsilon}\right|^{2} d x & =\int_{\partial \mathcal{H}_{z, \varepsilon}}\left[A\left(\nabla \varphi_{0}-\nabla \varphi_{\varepsilon}\right)\right]^{T} \cdot n \varphi_{0} d x \\
& =\frac{-2 \pi}{\log (\varepsilon)} \sqrt{|A|}\left|\varphi_{0}\left(x_{0}\right)\right|^{2}+o\left(\frac{-1}{\log (\varepsilon)}\right) .
\end{aligned}
$$

Hence

$$
\mathcal{J}\left(\Omega \backslash \overline{\mathcal{H}_{z, \varepsilon}}\right)-\mathcal{J}(\Omega)=D \mathcal{J}_{0}\left(\varphi_{0}\right)\left(\varphi_{\varepsilon}-\varphi_{0}\right)+\frac{-2 \pi \sqrt{|A|}}{\log (\varepsilon)}\left|\varphi_{0}(z)\right|^{2}+o\left(\frac{-1}{\log (\varepsilon)}\right) .
$$

Finally, by theorem 3.1 , we deduce

$$
\mathcal{J}\left(\Omega \backslash \overline{\mathcal{H}_{z, \varepsilon}}\right)-\mathcal{J}(\Omega)=\frac{-1}{\log (\varepsilon)} 2 \pi \sqrt{|A|}\left[\varphi_{0}(z) v_{0}(z)+\left|\varphi_{0}(z)\right|^{2}\right]+o\left(\frac{-1}{\log (\varepsilon)}\right)
$$

\section{The Kohn-Vogelius norms}

The Kohn-Vogelius criterion [15] is used like a cost functional. Since the boundary conditions $\left(\varphi_{d}, \Phi\right)$ are overspecified, one can define for any hole $\mathcal{H}$ two forward problems:

- the "Dirichlet" problem:

$$
\begin{cases}-\operatorname{div}\left(A \nabla \varphi_{D}\right)=0 & \text { in } \quad \Omega \backslash \overline{\mathcal{H}} \\ \varphi_{D}=\varphi_{d} & \text { on } \quad \Gamma_{d} \\ \varphi_{D}=0 & \text { on } \quad \partial \mathcal{H}\end{cases}
$$

- the "Neumann" problem:

$$
\begin{cases}-\operatorname{div}\left(A \nabla \varphi_{N}\right)=0 & \text { in } \quad \Omega \backslash \overline{\mathcal{H}}, \\ A \nabla \varphi_{N} \cdot \mathbf{n}=\Phi & \text { on } \quad \Gamma_{n}, \\ \varphi_{N}=0 & \text { on } \quad \partial \mathcal{H} .\end{cases}
$$

The optimal hole $\mathcal{H}^{*}$ coincides with the actual boundary $\mathcal{H}$ when the misfit between the solutions vanishes: $\varphi_{D}=\varphi_{N}$. Therefore, we propose an identification process based on the minimization of the following energy functional

$$
\mathcal{J}(\Omega \backslash \overline{\mathcal{H}})=\int_{\Omega \backslash \overline{\mathcal{H}}} A\left|\nabla \varphi_{N}-\nabla \varphi_{D}\right|^{2} d x
$$

This is the so-called Kohn-Vogelius criterion [15]. Our approach concerns the derived topological optimization problem:

$$
\min _{\mathcal{H} \subset \Omega} \mathcal{J}(\Omega \backslash \overline{\mathcal{H}})
$$


We will use the topological gradient method to solve this problem. It provides an asymptotic expansion of the function $\mathcal{J}$ with respect to a small topological perturbation of the domain $\Omega$.

5.1. Asymptotic expansion of the cost functional. The following Theorem describes the variation of the function $\mathcal{J}$ when creating a small hole $\mathcal{H}_{z, \varepsilon}$ inside the domain $\Omega$ with a Dirichlet boundary condition on $\partial \mathcal{H}_{z, \varepsilon}$.

$$
\text { For all } \varepsilon \geq 0, \mathcal{J}\left(\Omega \backslash \overline{\mathcal{H}_{z, \varepsilon}}\right)=\int_{\Omega \backslash \overline{\mathcal{H}_{z, \varepsilon}}} A\left|\nabla \varphi_{N}^{\varepsilon}-\varphi_{D}^{\varepsilon}\right|^{2} d x
$$

where $\varphi_{N}^{\varepsilon}$ and $\varphi_{D}^{\varepsilon}$ are the solutions to the systems

$$
\left\{\begin{array}{ll}
-\operatorname{div}\left(A \nabla \varphi_{N}^{\varepsilon}\right)=0 & \text { in } \quad \Omega \backslash \overline{\mathcal{H}_{z, \varepsilon}}, \\
A \nabla \varphi_{N}^{\varepsilon} \cdot \mathbf{n}=\Phi & \text { on } \quad \Gamma_{n}, \\
\varphi_{N}^{\varepsilon}=0 & \text { on } \quad \partial \mathcal{H}_{z, \varepsilon} .
\end{array} \quad ; \quad \begin{cases}-\operatorname{div}\left(A \nabla \varphi_{D}^{\varepsilon}\right)=0 & \text { in } \quad \Omega \backslash \overline{\mathcal{H}_{z, \varepsilon}} \\
\varphi_{D}^{\varepsilon}=\varphi_{d} & \text { on } \quad \Gamma_{d} \\
\varphi_{D}^{\varepsilon}=0 & \text { on } \quad \partial \mathcal{H}_{z, \varepsilon}\end{cases}\right.
$$

5.1.1. The three dimensional case. In this paragraph, we present the topological asymptotic expansion for the Anisotropic Laplace equations in the three dimensional case. In this case the fundamental solution of the anisotropic Laplace operator $E$ is given by

$$
E(x)=\frac{-1}{4 \pi \sqrt{|A|}|| A_{*} x||}, \quad \forall x \in \Omega .
$$

Theorem 5.1. Under the same hypotheses of theorem 3.1, the function $\mathcal{J}$ has the following asymptotic expansion

$$
\mathcal{J}\left(\Omega \backslash \overline{\mathcal{H}_{z, \varepsilon}}\right)-\mathcal{J}(\Omega)=\varepsilon\left\{4 \pi \sqrt{|A|}\left[\varphi_{N}^{0}(z) v_{N}^{0}(z)+\varphi_{D}^{0}(z) v_{D}^{0}(z)\right]+\delta \mathcal{J}(z)\right\}+o(\varepsilon)
$$

with

$$
\delta \mathcal{J}(x)=4 \pi \sqrt{|A|}\left\{\left|\varphi_{N}^{0}(x)\right|^{2}+\left|\varphi_{D}^{0}(x)\right|^{2}\right\}, \quad \forall x \in \Omega .
$$

5.1.2. The two dimensional case. In this paragraph, the result is obtained using the same technique described in the previous paragraph. The unique difference comes from the expression of the fundamental solution of the Anisotropic Laplace equations. In this case $E$ is given by

$$
E(x)=\frac{1}{2 \pi \sqrt{|A|}} \log \left(\left\|A_{*} x\right\|\right), \quad \forall x \in \Omega .
$$

Theorem 5.2. Under the same hypotheses of theorem 3.1, the function $\mathcal{J}$ has the following asymptotic expansion

$$
\mathcal{J}(\Omega \backslash \overline{\mathcal{H} z, \varepsilon})-\mathcal{J}(\Omega)=\frac{-1}{\log (\varepsilon)}\left\{2 \pi \sqrt{|A|}\left[\varphi_{N}^{0}(z) v_{N}^{0}(z)+\varphi_{D}^{0}(z) v_{D}^{0}(z)\right]+\delta \mathcal{J}(z)\right\}+o\left(\frac{-1}{\log (\varepsilon)}\right),
$$

with

$$
\delta \mathcal{J}(x)=2 \pi \sqrt{|A|}\left\{\left|\varphi_{N}^{0}(x)\right|^{2}+\left|\varphi_{D}^{0}(x)\right|^{2}\right\}, \quad \forall x \in \Omega
$$




\section{Numerical Result}

This section is concerned with some numerical investigations. We consider the bidimentional case and we present a fast and simple one-iteration identification algorithm. The unknown object $\mathcal{H}$ is identified using a level set curve of the topological gradient $\delta \mathcal{J}$. More precisely, the unknown object $\mathcal{H}$ is likely to be located at zone where the topological gradient $\delta \mathcal{J}$ is more negative.

\section{One-iteration algorithm: $[11,12]$}

- Solve the two problems $\left(\mathcal{P}_{N}^{0}\right)$ and $\left(\mathcal{P}_{D}^{0}\right)$ in initial domain $\Omega$,

- Compute the topological gradient function $\delta \mathcal{J}(x), x \in \Omega$,

- Determine the unknown object

$$
\mathcal{H}=\left\{x \in \Omega, \text { such that } \delta \mathcal{J}(x)<c_{\text {min }}\right\},
$$

where $c_{\text {min }}$ is a negative constant chosen in such a way that the cost function $\mathcal{J}$ has the most negative value.

Next, we will present some numerical simulations using the proposed algorithm. In Figure 1, we test our algorithm on circular shape. In Figure 2, we consider the case of an elliptical shape. In Figure 3, we can notice that, when the shape is non-regular, the reconstruction is quite efficient. In the case of non trivial shape, yet we applied a one-iteration algorithm, we obtain an interesting reconstruction result (see Figure $4)$.

The obtained result can serve as a good initial estimate for an iterative optimization process based on the shape derivative.

The considered model can be viewed as a prototype of a geometric inverse problem valid in many applications.

6.0.1. Reconstruction of circular-shaped objects. In this case, we test our procedure to detect an object having circular-shaped. We reconstruct in this case the object $\mathcal{H}$ described by a disk centered at $z=(2,0)$ with different radius: $r \in\{0.2,0.4,0.6\}$. The obtained results are illustrated in Figure 1. One can easily observe in Figure 1, the unknown object is located in the region where the topological sensitivity function $\delta \mathcal{J}$ is the most negative (red zone). The boundary of $\mathcal{H}^{*}$ is approximated by an iso-value curve. Our one-iteration algorithm gives an efficient reconstruction results for the different chosen sizes.

6.0.2. Reconstruction of ellipse-shaped objects. In this example, we reconstruct an object described by an ellipse inserted in the disc $\mathcal{D}=B((2,0), 1)$ and centered at $(2,0)$. We represent the results in Figure 2. In this case, we examine the numerical reconstruction of various ellipses having different directions and sizes. As one can observe in Figure 2, the boundary of the object is again detected and located in the zone where 
the topological gradient is most negative (red lines). Also, our one-iteration algorithm gives quite effecient reconstruction results for different chosen ellipse-shaped objects.

6.0.3. Reconstruction of geometry with corners. We tried to apply our proposed algorithm to detect more complicated geometry. Our objective is to reconstruct an object with corners. More precisely, we are trying to detect a square and rectangle shape. We can see in the Figure 3 (a), that the unknown square $\mathcal{H}^{*}$ is located in the zone where the topological gradient function $\delta \mathcal{J}$ is the most negative (red zone) and also its boundary is approximated by an iso-value curve. So here, our one-iteration algorithm detects the location and the shape of the square. But in the case of a rectangle shape (see Figure 3 (b)) the boundary $\mathcal{H}^{*}$ cannot be approached by any iso-value curve of the topological gradient function. We can remark in this case, that the one-iteration algorithm detects the zone containing the unknown geometry but the reconstruction result is not good.

6.0.4. Reconstruction of a non trivial-shaped objects. We apply now our proposed algorithm to detect a non trivial shapes. We can see in Figure 4 that the unknown shape $\mathcal{H}^{*}$ is located in the zone where the topological gradient function $\delta \mathcal{J}$ is the most negative (red iso-values) but we cannot approximate the boundary of $\mathcal{H}^{*}$ by any iso-value curve of the topological sensitivity function $\delta \mathcal{J}$. We can improve these reconstruction results by suggesting an iterative algorithm.

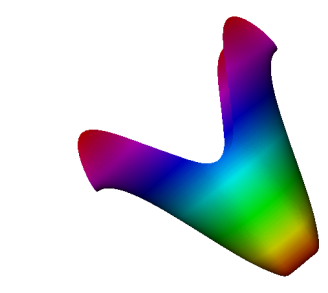

$\mathrm{r}=0.2$ :negative zone(red zone)

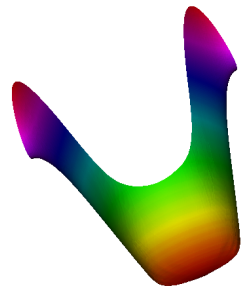

$\mathrm{r}=0.4$ :negative zone(red zone)

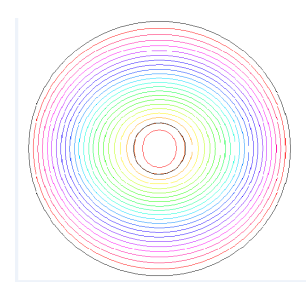

iso-value of $\delta \mathcal{J}$ approximating $\delta \mathcal{H}^{*}$ (black line)

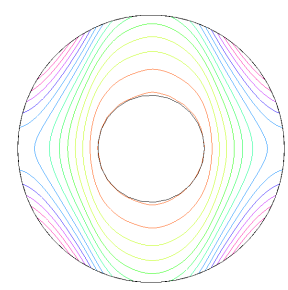

iso-value of $\delta \mathcal{J}$

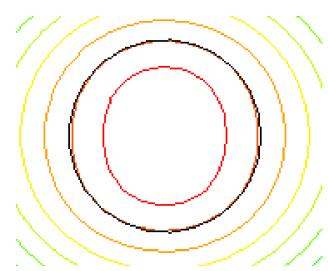

zoom showing the iso-value of $\delta \mathcal{J}$

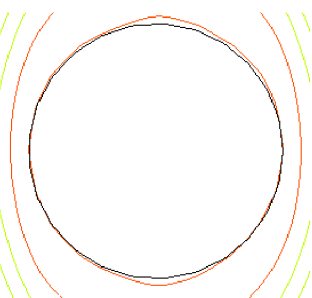

zoom showing the iso-value of $\delta \mathcal{J}$

approximating $\delta \mathcal{H}^{*}$ (black line) 


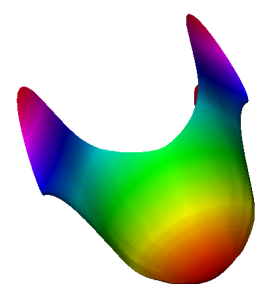

$\mathrm{r}=0.6$ :negative zone(red zone)

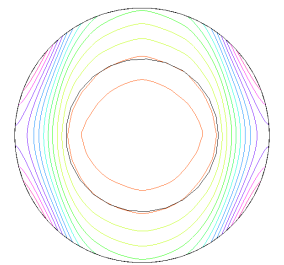

iso-value of $\delta \mathcal{J}$

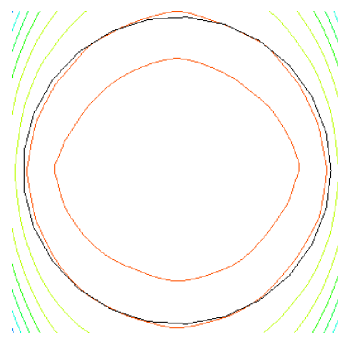

zoom showing the iso-value of $\delta \mathcal{J}$

approximating $\delta \mathcal{H}^{*}$ (black line)

FiguRE 1. Reconstruction of circle shaped objects
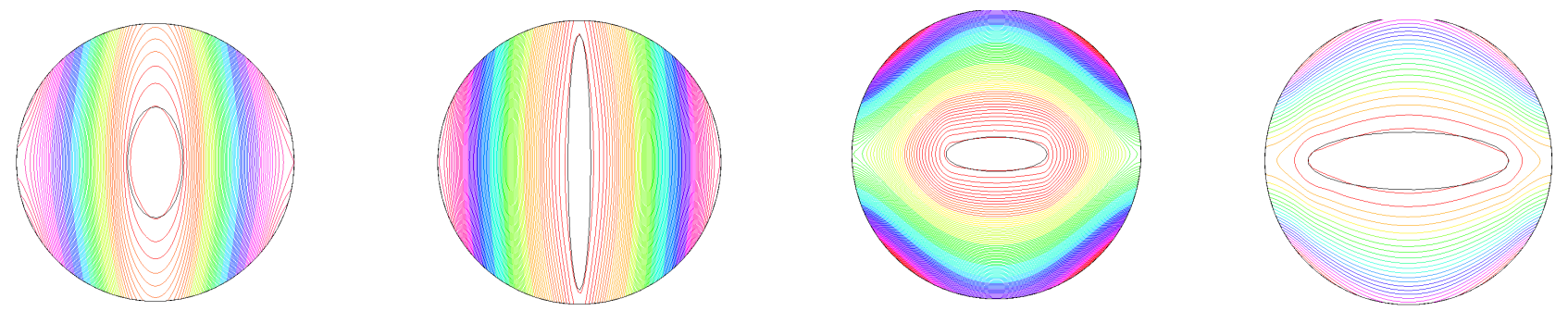

FIGURE 2. Reconstruction of an ellipse shaped objects

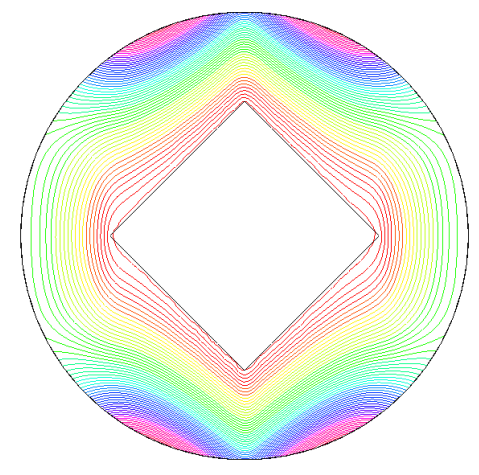

(a) square shape

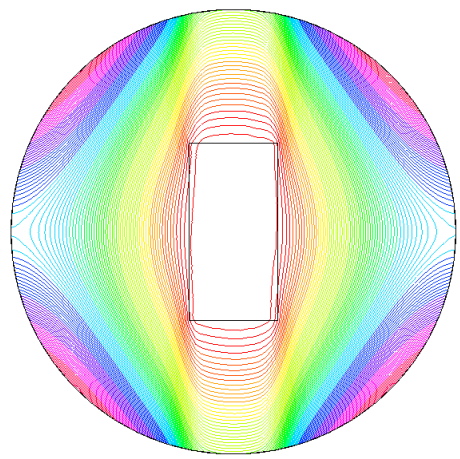

(b) rectangle shape

FIGURE 3. Reconstruction of objects with corners.

\section{ACKNOWLEDGEMENT}

The author gratefully acknowledge the approval and the support of this research study by the grant no. 7772-SCI-2018-3-9-F from the Deanship of Scientific Research at Northern Border University, Arar, Saudi Arabia. 


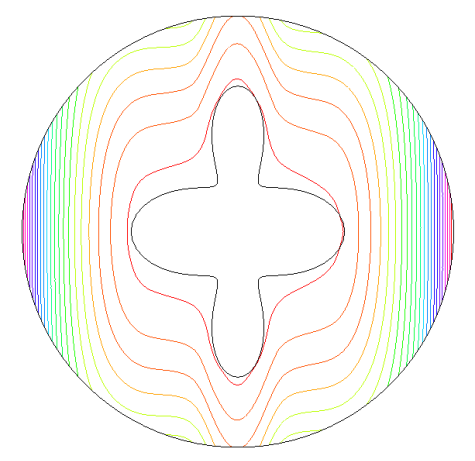

FIGURE 4. Reconstruction of a non trivial shape.

Conflicts of Interest: The author(s) declare that there are no conflicts of interest regarding the publication of this paper.

\section{REFERENCES}

[1] M. Abdelwahed, M. Hassine and M. Masmoudi, Optimal shape design for fluid flow using topological perturbation technique, J. Math. Anal. Appl. 356(2) (2009), 548-563.

[2] M. Abdelwahed and M. Hassine, Topological optimization method for a geometric control problem in stokes flow, Appl. Numer. Math. 59(8) (2009), 1823-1838.

[3] L. Afraites, M. Dambrine, K. Eppler and D. Kateb, Detecting perfectly insulated obstacles by shape optimization techniques of order two, Discrete Contin. Dyn. Syst. Ser. B, 8(2) (2007), 389-416.

[4] S. Amstutz, Aspects théoriques et numériques en optimisation de forme topologique, PhD thesis, Toulouse, INSA, 2003.

[5] S. Amstutz, The topological asymptotic for the navier-stokes equations, ESAIM: Control, Optim. Calc. Var. 11(3) (2005), 401-425.

[6] S. Andrieux, T. Baranger and A. Ben Abda, Solving cauchy problems by minimizing an energy-like functional. Inverse Probl. 22(1) (2006), 115-133.

[7] M. Badra, F. Caubet and M. Dambrine, Detecting an obstacle immersed in a fluid by shape optimization methods, Math. Models Meth. Appl. Sci. 21(10) (2011), 2069-2101.

[8] S. Garreau, P. Guillaume and M. Masmoudi, The topological asymptotic for pde systems: the elasticity case, SIAM J. Control Optim. 39(6) (2001), 1756-1778.

[9] P. Guillaume and K. Sid Idris, The topological asymptotic expansion for the dirichlet problem, SIAM J. Control Optim. 41(4) (2002), 1042-1072.

[10] A. Hamdi, A non-iterative method for identifying multiple unknown timedependent sources compactly supported occurring in a 2d parabolic equation,Inverse Probl. Sci. Eng. 26(5) (2018), 744-772.

[11] M. Hassine and I.Kallel, Kohn-vogelius formulation and topological sensitivity analysis based method for solving geometric inverse problems, Arab J. Math. Sci. 24(1) (2018), 43-62.

[12] M. Hassine and I. Kallel, One-iteration reconstruction algorithm for geometric inverse problems, Appl. Math. E-Notes, 18 (2018), 43-50. 
[13] L. Jackowska-Strumillo, J. Sokolowski and A. Zochowski, The topological derivative method in shape optimization, In: Proceedings of the 38th IEEE Conference on Decision and Control (Cat. No. 99CH36304), volume 1, pages 674-679. IEEE, 1999.

[14] E. Kim, H. Kang and K. Kim, Anisotropic polarization tensors and detection of an anisotropic inclusion, SIAM J. Appl. Math. 63(4) (2003), 1276-1291.

[15] R. V. Kohn and M. Vogelius, Relaxation of a variational method for impedance computed tomography, Commun. Pure Appl. Math. 40(6) (1987), 745-777.

[16] M. Hassine, Shape optimization for the stokes equations using topological sensitivity analysis, Rev. Afr. Rech. Inform. Math. Appl. 5 (2016), 216-229.

[17] M. Masmoudi, The topological asymptotic, computational methods for control applications, ed. H. Kawarada and J. Périaux. International Series, Gakuto, 2002.

[18] M. Masmoudi, J. Pommier and B. Samet, The topological asymptotic expansion for the maxwell equations and some applications, Inverse Probl. 21(2) (2005), 547.

[19] A. Novotny and J. Soko lowski, Topological derivatives in shape optimization, Springer Science \& Business Media, 2012.

[20] J. Pommier and B. Samet, The topological asymptotic for the helmholtz equation with dirichlet condition on the boundary of an arbitrarily shaped hole, SIAM J. Control Optim. 43(3) (2004), 899-921.

[21] B. Samet, S. Amstutz and M. Masmoudi, The topological asymptotic for the helmholtz equation, SIAM J. Control Optim. $42(5)$ (2003), 1523-1544.

[22] W. Yan, M. Liu and F. Jing, Shape inverse problem for stokes- brinkmann equations, Appl. Math. Lett. 88 (2019), $222-229$. 